\title{
Acute pyogenic psoas abscess in a child with nephrotic syndrome
}

\author{
*K Jagadish Kumar ${ }^{1}$, Joseph Solbin ${ }^{2}$, M G Anil Kumar ${ }^{3}$, C Anitha ${ }^{4}$ \\ Sri Lanka Journal of Child Health, 2016; 45(3): 226-228 \\ DOI: http://dx.doi.org/10.4038/sljch.v45i3.8146 \\ (Key Words: Nephrotic syndrome, peritonitis, psoas abscess)
}

\section{Introduction}

Nephrotic syndrome (NS) is a common kidney disease in children. Children with NS are immunocompromised, more susceptible to bacterial infections and primary peritonitis is a welldescribed complication ${ }^{1,2}$. Infections are the important cause of morbidity and mortality in nephrotic children ${ }^{1}$. The unique defence mechanisms of the peritoneal cavity promote localization resulting in the formation of abscesses $^{3}$. Even though peritonitis is a well described entity in NS, intra-abdominal abscesses are very rare 4 . A literature search did not reveal psoas abscess as a complication in NS. In children who present with a limp or lower abdominal pain, psoas abscess does not head the list in the differential diagnosis 5 . We report a psoas abscess in a child with NS who presented with lower abdominal pain and limping of the right leg.

\section{Case report}

A 6 year old girl with NS presented with lower abdominal pain, vomiting and limping of the right leg since one week. There was no history of fever or trauma. She was on alternate day treatment with prednisolone $1.5 \mathrm{mg} / \mathrm{kg}$ since the last 3 months. On examination, she was afebrile with a respiratory rate of 28/minute, pulse rate of $120 /$ minute and blood pressure of $120 / 72 \mathrm{~mm} \mathrm{Hg}$. Her abdominal examination revealed a distended abdomen with severe tenderness in the right iliac fossa and right lumbar region associated with guarding on the right side of the abdomen. Hip joint movements were painful and restricted. Other systems examination was unremarkable. In view of the severe tenderness of the right side of the abdomen with painful and

\footnotetext{
${ }^{1}$ Professor of Paediatrics, ${ }^{2}$ Resident in Paediatrics, ${ }^{3}$ Professor of Paediatric Surgery, ${ }^{4}$ Associate Professor of Paediatrics, JSS Medical College, JSS University, Mysore, India

*Correspondence: jagdishmandya@gmail.com (Received on 10 February 2015: Accepted after revision on 20 March 2015)
}

The authors declare that there are no conflicts of interest

Personal funding was used for this project.

Open Access Article published under the Creative

Commons Attribution CC-BY (CC) restricted hip joint movements in an immunocompromised child probable diagnoses of peritonitis, acute appendicitis or septic arthritis of the right hip joint were entertained.

The haemoglobin was $11.6 \mathrm{~g} / \mathrm{dl}$, total leucocyte count 31,730/ cu mm (N 91\%, L7\%) and the platelet count $406,000 / \mathrm{cu} \mathrm{mm}$. Kidney function tests were normal. Liver function tests were normal except for a total protein of $4.1 \mathrm{~g} / \mathrm{L}$ and a serum albumin of $1.4 \mathrm{~g} / \mathrm{L}$. Her urine showed 3-plus albumin and blood culture was sterile. Her chest $\mathrm{x}$ ray, pelvis $\mathrm{x}$-ray and lumbar spine $\mathrm{x}$-ray were normal. Her Mantoux test, Anti Human Immunodeficiency Virus antibodies and Hepatitis $B$ surface antigen were negative.

Sonography revealed a large loculated collection with septae in right iliac fossae extending up to right lumbar region measuring $6 \times 7 \mathrm{~cm}$ with mild ascites (Figure 1).

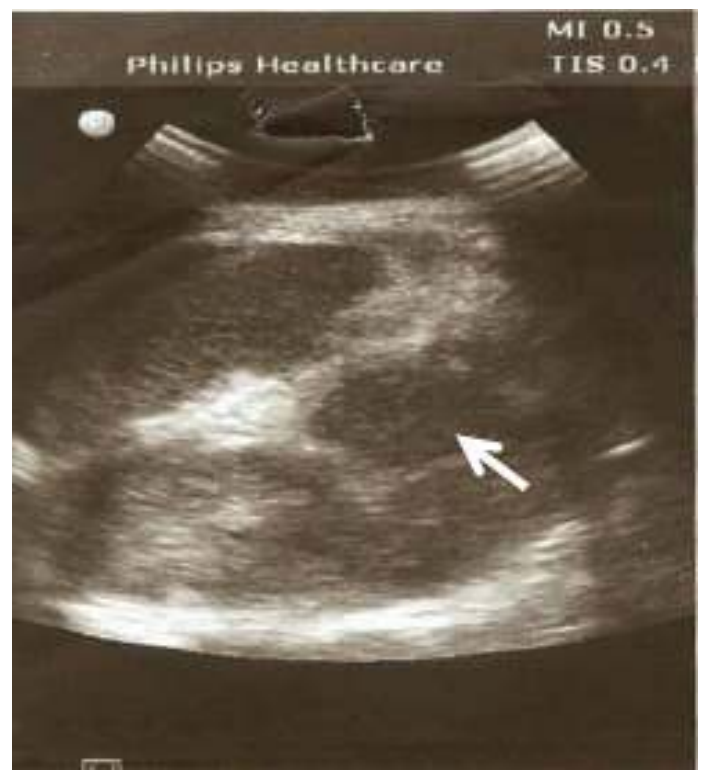

Figure 1: Sonography showing a large loculated collection

Sonography of the right hip joint was normal. She was started on injection ceftriaxone, linezolid and metronidazole. Abdominal laparotomy was done and $200 \mathrm{ml}$ of pus was drained from right psoas abscess which was extending up to pelvic cavity. Pus culture grew staphylococcus capitis, sensitive 
to ciprofloxacin, ofloxacin, clindamycin, linezolid, vancomycin and trimethoprim-sulfamethoxazole. Within 24 hours of starting intravenous antibiotics, her pain subsided and she was discharged after 14 days of therapy.

\section{Discussion}

Children with NS are immuno-compromised and therefore are exposed to a variety of infections resulting in morbidity and mortality ${ }^{1}$. Primary peritonitis is a well-described, alarming infectious complication and has a reported incidence of 1.5 $16 \%$ in $\mathrm{NS}^{2}$. Children with NS are immunocompromised due to hypoproteinaemia particularly hypogammaglobulinaemia, decreased complement factors $\mathrm{B}$ (C3 proactivator) and $\mathrm{D}$. There is also evidence of abnormal T-lymphocyte function, hypovolaemia resulting in hypoperfusion of spleen and intestinal mucosa often leading to hypercoagulation and micro-infarcts with oedematous tissue behaving as a culture medium for bacterial growth. Immunosuppressive effects of steroids may also contribute to this ${ }^{2}$. Intra-abdominal abscesses form one extreme in the spectrum of bacterial peritonitis ${ }^{3}$. Even though peritonitis is a well described entity in NS, intra-abdominal abscesses are very rare. To the best of our knowledge only a single case of primary retroperitoneal abscess has been reported so far in $\mathrm{NS}^{4}$.

Psoas abscess is a collection of pus in the iliopsoas compartment and is caused by the contiguous spread from infected organs or by haematogenous spread from sites of occult infection aided by the rich vascular supply of muscle ${ }^{6}$. Psoas abscesses in children are rare and may be classified as primary or secondary ${ }^{7}$. Primary psoas abscess is one without obvious focus of infection and secondary psoas abscess often occurs as a direct spread from contiguous structures ${ }^{8}$. In children it is usually primary as it was in our child ${ }^{7}$. The clinical presentation of iliopsoas abscess is often variable and non-specific ${ }^{6}$. Because of its relative infrequency and non specific presentation there will be a delay in diagnosis and treatment ${ }^{6,9}$. It is often an overlooked clinical entity and psoas abscess does not head the list in the differential diagnosis of the child who presents with a limp or lower abdominal pain ${ }^{5,8}$. The classical clinical triad of psoas abscess includes fever, back pain, and limp ${ }^{6}$. Our child presented with lower abdominal pain and limp but without fever, probably due to long term steroids. A common clinical sign is a positive psoas sign (pain when the hip is passively extended or actively flexed against resistance), which is attributed to inflammation leading to spasm of the psoas muscle. This was present in our child and was mistakenly attributed to right hip joint septic arthritis ${ }^{7}$. The original admitting diagnosis in all 7 cases of psoas abscesses in children was septic arthritis of the hip in a study by Schwaitzberg et $\mathrm{al}^{5}$. In a study of 24 children with psoas abscesses, the main initial complaints were a painful hip and difficulty in walking ${ }^{10}$.

Staphylococcus aureus is the most frequently cultured organism in over $88 \%$ of patients with primary iliopsoas abscess ${ }^{6,7,8}$. However, in our child, pus from the psoas abscess grew Staphylococcus capitis i.e. coagulase negative staphylococci. Staphylococcus aureus was isolated in 20 out of 24 children with psoas abscess ${ }^{10}$.

Psoas abscess is easily diagnosed by ultrasonography ${ }^{8,9}$. Sonography is accurate, inexpensive and is easy to perform without radiation effects ${ }^{8}$. In a study by Kadambari et al, ultrasound confirmed the diagnosis in all 36 cases of psoas abscess and CT was not done in any of the them $^{8}$. Ultrasonography was used as a screening method to confirm the clinical diagnosis in 19 children with psoas abscess ${ }^{10}$. CT is considered the gold standard for diagnosis as the retroperitoneal space may be difficult to visualise ultrasonographically and can also be obscured by bowel gas ${ }^{6}$. Early surgical drainage with appropriate antibiotics has been the standard method of treatment and our child responded well following the drainage procedure $e^{7,8}$.

A high index of suspicion of psoas abscess is mandatory when dealing with intra-abdominal complications in children with NS, especially when they present with fever, limping and lower abdominal pain and sonography should always be done as it is a valuable diagnostic tool.

\section{References}

1. Tain YL, Lin GJ, Cher TW. Microbiological spectrum of septicaemia and peritonitis in nephrotic children. Pediatric Nephrology1999; 13:835-7. http://dx.doi.org/10.1007/s004670050710 PMid: 10603131

2. Uncu N, Bülbül M, Yildiz N, Noyan A, Kosan C, Kavukçu S et al. Primary peritonitis in children with nephrotic syndrome: results of a 5-year multicentre study. European Journal of Pediatrics 2010; 169:73-6. http://dx.doi.org/10.1007/s00431-0090989-x

PMid: 19430812

3. Thompson AE, Marshall JC, Opal SM. Intra-abdominal infections in infants and children: Descriptions and definitions. Pediatric Critical Care Medicine 2005; 6[Suppl.]:S30 -S35. 
http://dx.doi.org/10.1097/01.PCC.000016

1963.48560.55

PMid: 15857555

4. Russo GA, Lichtner MA, Carnevalini MA, Mascellino MT, Mengoni FA, Oliva AA, et al. Primary retroperitoneal abscesses due to Rhodococcus equi in a patient with severe nephrotic syndrome: successful antibiotic treatment with linezolid and tigecycline. International Journal of Infectious Diseases 2010; 14:533-5. http://dx.doi.org/10.1016/j.ijid.2009.06.02 1

PMid: 19758832

5. Schwaitzberg SD, Pokorny WJ, Thurston RS, McGill CW, Athey PA, Harberg FJ. Psoas abscess in children. Journal of Pediatric Surgery 1985; 20:339-42. http://dx.doi.org/10.1016/S00223468(85)8 0215-3

6. Mallick IH, Thoufeeq MH, Rajendran TP. Iliopsoas abscesses Postgraduate Medical Journal 2004; 80:459-62.

http://dx.doi.org/10.1136/pgmj.2003.0176 65

PMid: 15299155 PMCid: PMC1743075
7. Atkinson $\mathrm{C}$, Morris $\mathrm{SK}$, Vicky $\mathrm{Ng}$, Friedman JN. A child with fever, hip pain and limp. Canadian Medical Association Journal 2006; 174(7): 924. http://dx.doi.org/10.1503/cmaj.051165 PMid: 16567754 PMCid: PMC1405870

8. Kadambari D, Jagdish S. Primary pyogenic psoas abscess in children. Pediatric Surgery International 2000; 16:408-10.

http://dx.doi.org/10.1007/s003839900329 PMid: 10955575

9. Bresee JS, Edwards MS. Psoas abscess in children. Pediatric Infectious Disease Journal 1990; 9:201-6. http://dx.doi.org/10.1097/0000645419900 3000-00011

10. Parbhoo A, Govender S. Acute pyogenic psoas abscess in children. Journal of Pediatric Orthopaedics 1992; 12:663-6. http://dx.doi.org/10.1097/0124139819920 9000-00019

PMid: 1517431 